\title{
Pharmacological and non-pharmacological interventions improve food intake in cancer related anorexia and cachexia
}

Brown JK. A systematic review of the evidence on symptom management of cancer-related anorexia and cachexia. Oncol Nurs Forum 2002 Apr;29:517-32.

\section{QUESTION: What is the effectiveness of various strategies for management of cancer related anorexia and cachexia?}

\section{Data sources}

Studies were identified by searching the Cochrane Library, Medline, CANCERLIT, CINAHL, Dissertation Abstracts, EBM Reviews-Best Evidence, EMBASE/ Excerpta Medica, and the Computer Retrieval of Information on Scientific Projects (CRISP).

\section{Study selection}

Clinical trials were selected if the focus was to increase food intake, decrease energy expenditure, minimise weight loss, or minimise factors affecting food intake or energy expenditure in patients with cancer. Studies of micronutrient supplementation over recommended daily allowances and alternative dietary cancer treatments were excluded.

\section{Data extraction}

Data were extracted on study design and setting, sample and follow up, intervention and adherence measures, outcome measures, and analysis.

\section{Main results}

7 clinical trials of non-pharmacological interventions, 4 reviews of pharmacological interventions and exercise, 1 meta-analysis of nursing symptom management, and 3 general overviews met the selection criteria. Nonpharmacological interventions: 7 randomised controlled trials (RCTs) (n=558) compared nutritional counselling and/or commercial oral liquid supplements with various control interventions. All 7 studies reported improved caloric intake with nutritional counselling and oral liquid supplementation. No differences were found for survival, tumour response, or nutritional status. 4 of 5 studies found no differences for weight loss, and 1 study $(\mathrm{n}=26)$ reported that progressive weight loss was stopped in patients with advanced cancer who received nutritional counselling and liquid oral supplementation. 2 of 2 studies found no differences for quality of life. Pharmacological interventions: 3 reviews addressed the effectiveness of 4 drug classes: progestational agents (megestrol acetate and medroxyprogesterone), corticosteroids, hydrazine, and cyproheptadine. Megestrol acetate, $160-1600 \mathrm{mg} /$ day, improved appetite, caloric intake, body weight (mostly fat), and sensation of wellbeing (11 placebo controlled studies). Studies of medroxyprogesterone acetate, 300$1000 \mathrm{mg} /$ day, showed similar results. Both drugs commonly caused oedema as a side effect. A review of 5 randomised placebo controlled trials found that corticosteroids improved appetite, food intake, performance, and quality of life in the short term ( $\leq 4 \mathrm{wks})$, but did not affect weight. This review also identified 6 placebo controlled trials of hydrazine and reported that recent large placebo controlled trials found no increases in body weight, but did find toxicity and a decline in quality of life. 1 placebo controlled trial $(\mathrm{n}=295)$ found that cytoheptadine resulted in small increases in appetite, food intake, and sedative effects. Physical activity interventions: in a review of 18 small studies (10 quasi-experimental and 8 experimental, mean
25 participants/study), 16 studies found that aerobic exercise improved quality of life, functional capacity, muscle strength, body composition, and haematological indicators, and reduced nausea, fatigue, diarrhoea, anxiety, and depression.

\section{Conclusion}

In patients with cancer related anorexia and cachexia, both non-pharmacological and pharmacological interventions increase food intake. Selected pharmacological interventions improve appetite, sensation of wellbeing, and body weight, but do not improve body compostion or nutritional status.

\section{COMMENTARY}

Anorexia, the loss of appetite or desire to eat, is one of the most common symptoms in patients with cancer. It can occur early in the disease process or later as the tumour grows and metastasises. Anorexia occurs in 30-79\% of patients with metastatic disease. ${ }^{1}$ The systematic review by Brown examined studies addressing management of cancer related anorexia and cachexia (weight loss and muscle wasting), separating pharmacological from nonpharmacological approaches. The clinical application of the study findings requires an integrated approach, in which patient goals may be restorative or palliative, and relevant to the individual patient at a particular point of his/her disease.

This type of descriptive systematic review provides problems of interpretation. The 7 non-pharmacological studies included in the review examined a variety of outcome measures, from tumour response and survival to nutritional status and quality of life. These differing outcomes, combined with the methodological weaknesses of individual trials, make it difficult to draw conclusions about those interventions commonly used by nurses. The strongest body of evidence shows that the drugs megestrol acetate and medroxyprogesterone increase wellbeing, appetite, and weight gain, but do not improve body composition or nutritional status. A remarkable finding of the review is that the effect of oral nutritional supplements (widely promoted by nurses in cancer care) has not been properly evaluated. It is also clear from the review that there is a lack of consensus amongst researchers and clinicians regarding the goal of anorexia and cachexia management. The most commonly measured outcomes among the non-pharmacological studies were tumour response and survival; data on quality of life and patient wellbeing were sparse. Brown is right to argue for more research on multifaceted approaches to the management of anorexia and cachexia. The use of progestationals and corticosteroids provide positive benefit. Nutritional strategies, physical activity, and other supportive care approaches may optimise the management of anorexia and cachexia in an oncology setting, but further research is needed.

Pamela Piotrowski, BASc, RD

Clinical Dietitian, Hamilton Regional Cancer Centre Hamilton, Ontario, Canada 